\title{
Orbital Apex Syndrome Resulting from Mixed Bacterial Sphenoid Sinusitis
}

\author{
Mengfei Xiong ${ }^{1}$, Wai Lun Moy ${ }^{2}$ \\ ${ }^{1}$ Ministry of Health Holdings, Singapore \\ ${ }^{2}$ Sengkang General Hospital, Singapore
}

\section{Received: $19 / 04 / 2018$}

Accepted: 20/04/2018

Published: 22/05/2018

How to cite this article: Xiong M, Moy WL. Orbital apex syndrome resulting from mixed bacteria sphenoid sinusitis. EJCRIM 2018;5: doi:10.12890/2018_000905.

Conflicts of Interests: The Authors declare that there are no competing interests.

Acknowledgements: We thank our radiology colleagues Dr Jagadish N. Shenoy, Dr Dinesh R. Singh and Dr Low Tze-Anns' Keynes for providing the relevant analysis reports and images which greatly assisted our report.

This article is licensed under a Commons Attribution Non-Commercial 4.0 License

\section{ABSTRACT}

Orbital apex syndrome (OAS) is an uncommon disorder characterized by visual loss, ophthalmoplegia, ptosis and hypoaesthesia of the forehead ${ }^{[1]}$. OAS may result from a variety of inflammatory, infectious, neoplastic and vascular conditions that cause damage to the superior orbital fissure (with resultant oculomotor (III), trochlear (IV), abducens (VI) and ophthalmic branch of the trigeminal nerve (V1) palsies) and to the optic canal leading to optic nerve (II) dysfunction. This case report describes the clinical development of OAS in a patient with bacterial sphenoid sinusitis.

\section{LEARNING POINTS}

- Orbital apex syndrome (OAS) is an uncommon manifestation of a wide range of disease entities, with management ranging from antibiotic therapy to immunosuppression and surgery.

- OAS can be life-threatening if there is disease invasion through ophthalmic vessels or bone fissures, leading to intra-cranial involvement.

- Without adequate knowledge and clinical suspicion, OAS can be easily missed or misdiagnosed, resulting in delayed treatment and devastating loss of function or even death.

\section{KEYWORDS}

Orbital apex syndrome, superior orbital fissure, optic canal, optic nerve, type II diabetes mellitus, sphenoid sinusitis

\section{CASE DESCRIPTION}

A 58-year-old man with poorly controlled type II diabetes mellitus (HbA1c 8.2\%) was treated in hospital for right herpes zoster ophthalmicus and methicillin-sensitive Staphylococcus aureus (MSSA) osteomyelitis of his right fifth toe. He had completed his course of intravenous acyclovir and was close to finishing his treatment with intravenous cefazolin when he reported sudden onset of binocular diplopia. Examination at onset revealed isolated a 50\% reduction in left eye abduction; the right eye was normal.

Magnetic resonance imaging (MRI) of the brain showed significant abnormality. The images incidentally demonstrated mucosal thickening in the left posterior ethmoid and left sphenoid sinuses with retention cysts (Fig. 1B). Nasoendoscopy excluded nasopharyngeal carcinoma (NPC), but diagnosed left sphenoid sinusitis with possible orbital apex involvement. The patient was continued on intravenous cefazolin for his presumed bacterial sinusitis.

The patient developed partial ptosis 2 days later and the visual acuity of his left eye dropped from 6/7.5 to counting fingers. His pupils were 


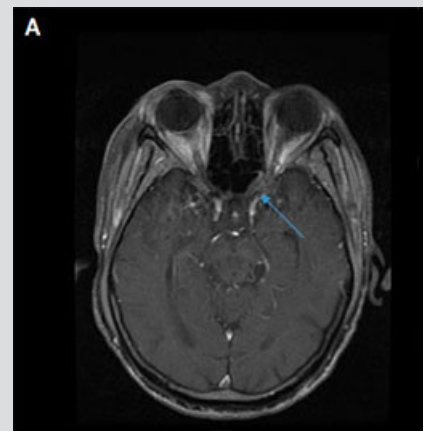

B
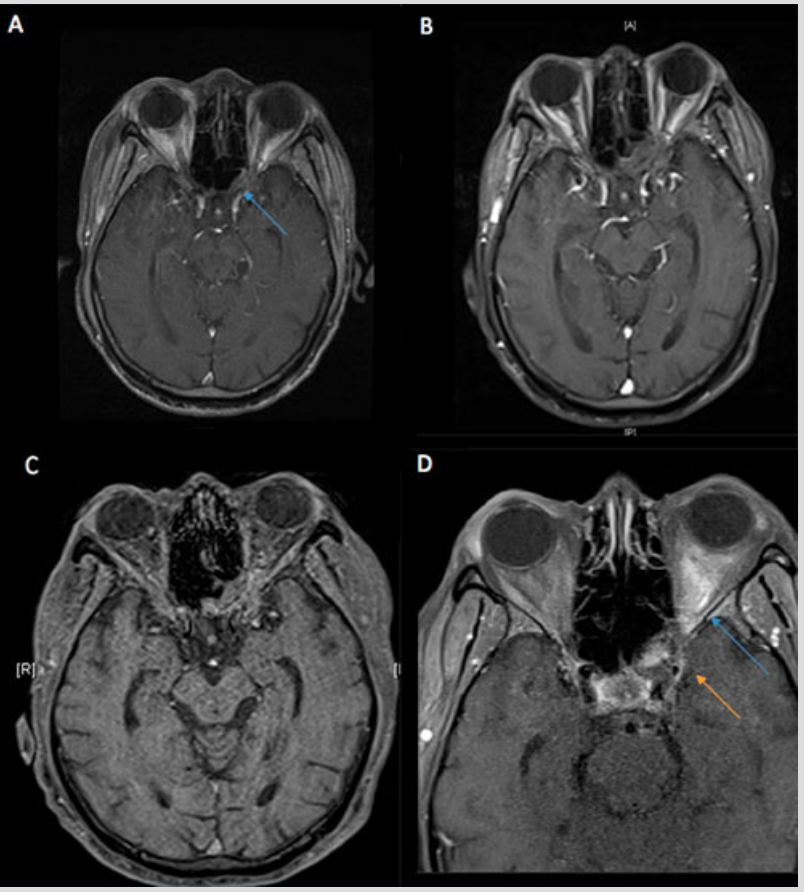

Figure 1. MRI brain images showing progression of orbital apex involvement.

(A) The initial scan on 29 August 2016 before the onset of neurological symptoms reveals only minor mucosal thickening (arrow) in the left sphenoid sinus.

(B) MRI of the brain on 16 September 2016 shortly after the onset of diplopia shows worsening of posterior ethmoid and sphenoid sinusitis, with slight prominent enhancement in the left orbital apex, which can be better appreciated on its T1W image (C).

(D) MRI of the orbit on 22 September 2016 demonstrates inflammatory soft tissue (orange arrow) extending beyond the left orbital apex into the retro-orbital region, with enhancement (blue arrow) of the rectus muscles and optic nerve

$2 \mathrm{~mm}$ reactive bilaterally with a positive corneal reflex. Fundoscopy showed no evidence of papilloedema. Shortly afterwards, there was near total ptosis of the left eye with progressive paresis of the remaining extra-ocular eye movements consistent with combined cranial nerve III, IV and VI palsies. Subsequently, visual acuity fell further to hand movements and relative afferent pupillary defect was positive on the left side. The remaining examination was normal.

Blood investigations including inflammatory markers were unremarkable during the development of neurological signs, and the patient tested negative for human immunodeficiency virus (HIV). Magnetic resonance thin-slice imaging of the orbit was performed, demonstrating the presence of soft tissue in the left sphenoid sinus extending towards the left orbital apex and anterior left cavernous sinus (Fig. 1D). The enhancement of retro-orbital fat, the rectus muscles and the left optic nerve were suggestive of significant intra-orbital inflammation similar to Tolosa-Hunt syndrome or fungal infection.

Computed tomography (CT) of the sinuses with an image-guided surgery protocol demonstrated left sphenoid and posterior ethmoid sinus mucosal thickening, with bony erosion of the left lateral wall of the sphenoid sinus, adjacent to the left orbital apex (Fig. 2).

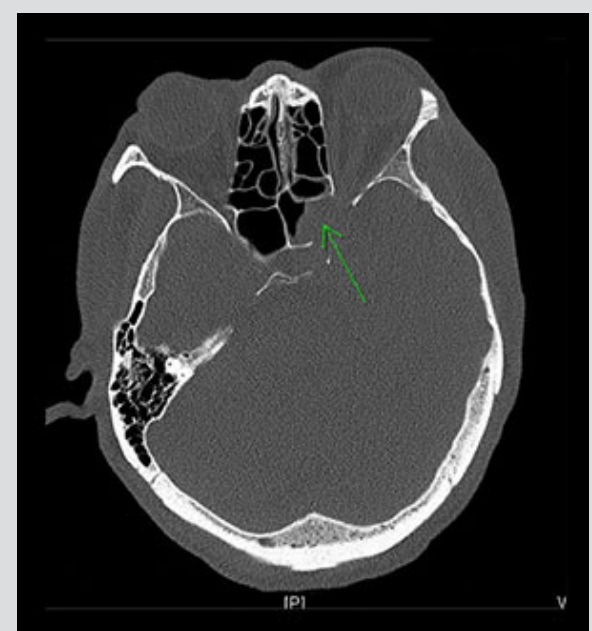

Figure 2. Green arrow indicating bony erosion of the left lateral wall of the sphenoid sinus. 
Following $\mathrm{CT}$, the patient underwent left sphenoidotomy with drainage of the sphenoid contents, the intra-operative cultures of which yielded methicillin-resistant Staphylococcus aureus (MRSA) and Pseudomonas aeruginosa, but which were negative for fungus and acid-fast bacilli. Histology revealed granulation tissue with acute and chronic inflammation.

The final diagnosis of bacterial sphenoid sinusitis with orbital apex involvement was established and treatment with intravenous vancomycin and cefepime was initiated. Unfortunately, the patient died from multi-organ failure before neurological improvement could be observed.

\section{DISCUSSION}

Initially our patient was treated with IV cefazolin alone, which provides sufficient coverage for most pathogens found in sinusitis; however, the rapid progression of clinical signs despite ongoing antibiotic therapy also raised suspicions of invasive fungal sinusitis. Although bacteria rarely cause invasive sinusitis, the rapid progression in this case may have been partly caused by our patient's poorly controlled diabetes and the antibiotic resistance of the causative bacteria. While both MRSA and P. aeruginosa are common pathogens in hospital-acquired infections, they do not usually cause nosocomial sinusitis, which is mainly seen in intensive care patients with nasal colonization by gramnegative bacilli or receiving enteral feeding via a nasogastric tube. Vancomycin was not initially started for our patient as he screened (nose, groin and axillary swabs) negative for MRSA on three separate occasions.

Among patients with orbital apex syndrome secondary to sinusitis, it has been found that those who have not yet developed radiographic findings of an orbital apex mass have a better prognosis and lower incidence of vision loss ${ }^{[2]}$. Therefore, when imaging reveals signs of mucosal invasion, early surgical debridement and directed antibiotic therapy is crucial to prevent irreversible loss of vision.

In addition to common causes such as infection and malignancy, the following rare conditions were also considered during the evaluation of our patient.

The first condition to be considered was Tolosa-Hunt syndrome, which is characterized by recurrent painful ophthalmoplegia while rarely affecting visual acuity. Diplopia is most frequently due to oculomotor (85\%) or abducens (70\%) neuropathy ${ }^{[3]}$. MRI images typically demonstrate a soft tissue mass lesion that enhances with contrast ${ }^{[4]}$. A significant reduction in size after corticosteroid therapy is particularly useful for diagnosis; hence it is essential to exclude infectious causes prior to initiation of treatment. In this case, although the MRI findings had features suggestive of Tolosa-Hunt syndrome, the involvement of the optic nerve as well as the absence of pain ruled out this diagnosis. The other conditions considered were immune-mediated systemic inflammatory disorders, for example immunoglobulin G4 (IgG4)-related disease. Patients with lgG4 disease usually present subacutely because of the slow development of orbital pseudotumor and lacrimal gland enlargement that lead to proptosis of the affected eye. The majority of cases of IgG4 disease with sinus involvement are also non-erosive lesions ${ }^{[5]}$, in contrast to the significant sphenoid wall erosion noted in this patient. Histological diagnosis can be made when classic findings such as dense lymphoplasmacytic infiltrate, storiform fibrosis and obliterative phlebitis are present. Immunohistochemical staining of biopsy specimens will also reveal elevated numbers of IgG4-positive plasma cells. None of these findings were present in this case.

\section{REFERENCES}

1. Yeh S, Foroozan R. Orbital apex syndrome. Curr Opin Ophthalmol 2004;15:490-498.

2. Colson AE, Daily JP. Orbital apex syndrome and cavernous sinus thrombosis due to infection with Staphylococcus aureus and Pseudomonas aeruginosa. Clin Infect Dis 1999;29:701-702

3. Lasam G, Kapur S. A rare case of Tolosa-Hunt-like syndrome in a poorly controlled diabetes mellitus. Case Rep Med 2016;2016:9763621.

4. Barnard B, Hurter D, Roux F, Aboobaker S. Tolosa-Hunt syndrome. S Afr J Radiol 2012;16:14-15.

5. Byrne TN, Stone JH, Pillai SS, Rapalino O, Deshpand V. Case 31-2016: a 53-year-old man with diplopia, polydipsia, and polyuria. N Engl J Med 2016;375:1469-1480. 